\title{
Multitasking: incentivizing agents differing either in their work ethic or intrinsic motivation
}

\author{
Carsten Helm ${ }^{1} \cdot$ Franz Wirl $^{2}$
}

Received: 10 October 2019/Accepted: 16 July 2020/Published online: 1 August 2020

(C) The Author(s) 2020

\begin{abstract}
Saint Thomas Aquinus 'agen autem non movet nisi ex intentione finis (an agent does not move except out of intention for an end, quoted from Nassim Nicholas Taleb, Antifragile, p. 169.)'. This paper uses the familiar multitasking framework in order to compare contracting with agents holding private information either about their work ethic or intrinsic motivation. Those characterizations are observation equivalent in the absence of incentives but matter once monetary incentives are offered. Indeed the difference is stark: First, incentives change the characterization of which types are efficient or inefficient. Second, contracts in terms of an agent's work ethic are robust if constraints (ensuring sufficient effort for the unobservable task) are introduced while such constraints can render only fixed wages feasible for intrinsically motivated agents.
\end{abstract}

Keywords Principal-agent model · Observation-equivalent out-of-contract $\cdot$ Multitasking - Work ethic - Intrinsic motive

JEL Classification D86 · J33

Franz Wirl

franz.wirl@univie.ac.at

Carsten Helm

carsten.helm@uni.oldenburg.de

1 Department of Economics and Law, University of Oldenburg, 26111 Oldenburg, Germany

2 Faculty of Business, Economics and Statistics, University of Vienna, Oskar Morgenstern Platz 1, Room 4.635, 1090 Wien, Austria 


\section{Introduction}

Managing multitasking is a widely acknowledged problem. Indeed, the contribution of Bengt Holmström was a major justification for awarding him the Nobel prize in 2016. An agent's positive contribution to an unobservable and, therefore, not incentivized task requires an intrinsic motive. As known since the seminal paper of Holmstrom and Milgrom (1991), incentivizing an agent with respect to an observable output will lower the agent's effort on the unobservable task and thus will impose costs beyond the usual agency costs. The IT-business provides an example where intrinsic motives are important. Fulfilling use-needs, personal enjoyment and satisfying a quest for learning (Benbya and Belbaly 2010) stimulate individuals up to the point of working free of charge in non-traditional production communities like Wikipedia, Linux and Mozilla (see e.g., Lakhani and Wolf 2005 on what motivates hackers and more recently Kolbjørnsrud 2016 accounting for agency problems in such communities). Recently, Goes et al. (2018) find that multitasking lowers customer satisfactions with firms offering consumer service chats enabled by information technology.

Our objective is to compare incentives, which are offered to agents who differ about what they hold as private information: either about their work ethic, which reduces their effort cost, or their intrinsic motivation, i.e. their private benefit from the non-observable task. This differentiation is close to the application of the multitasking framework to bonus culture in Benabou and Tirole (2016). Our characterizations of types imply the same performance in the absence of incentives and are in this sense observation-equivalent. This property is the only reason for choosing work ethic instead of productivity as Benabou and Tirole (2016). Moreover, work ethic and productivity are equivalent in the case of a single task with and without incentives. We are interested whether the two observationequivalent types receive different incentives and, how they differ in terms of efforts, wages, employment and output. We also examine under which circumstances incentives are feasible, and which types particular firms might actively seek.

We find that very similar and in the above sense even equivalent characterizations lead to the following surprising results:

1. Incentivizing leads to different contracts and the difference can be substantial.

2. The order of efficiency (in terms of the tasks prescribed and of the point of no distortion from the first best) is reversed for intrinsically motivated types, i.e., the lowest type is asked to deliver the highest output, although high types are more efficient under a fixed wage.

3. Sufficient importance of the unobservable task constrains what the principal can demand in terms of the observable task. Adding this binding constraint still allows for incentives if the agents are characterized by their work ethic but can render incentives impossible for intrinsically motivated agents.

The first point is not confined to multitasking and is a general warning far beyond the particular case. It applies to all applications in which the agents' utility function is additively separable — e.g., to represent a cost-benefit trade-off — so that one has to 
decide to which term the private information should be attached. Although observation-equivalence seems to suggest that one can choose between attaching the private information to either term, this is not the case so that this choice must be exercised with reason and care.

The other two points are specific to multi-tasking. The reversing of the ordering of an agent's efficiency is puzzling because the principal prefers high types under no incentives for both characterizations and also if incentivizing agents differing with respect to their work ethic. Clearly, a high work ethic allows for higher demands of the observable output and only this output can be incentivized. A high intrinsic motive of providing the necessarily unincentivized unobservable task makes it harder to incentivize the observable task. This makes high types less attractive for a principal using incentives.

The reason for the third point-the impossibility to incentivize agents characterized by their intrinsic motive-is linked to the above explanation. The necessity of a minimal contribution to the unobservable task constrains what the principal can demand from the observable task. Given the observation-equivalence, the same observable output results contingent on the unobservable one and is increasing with respect to both types, work ethic and intrinsic motivation. However, the interior mechanism is a declining one for intrinsically motivated agents for the above given reason. Combining the declining interior with the increasing boundary solution violates the requirement of monotonicity and thus renders incentives impossible. In contrast, agents characterized by their work ethic can be incentivized even if a contract must ensure a minimal output of the unobservable task. This has the empirically testable implication that the market should provide incentives in one case (work ethic) but much less so in the other case (intrinsic motivation). A further consequence is that the introduction of incentives due to liberalization, more (including international) competition and in the extreme case of a transition from socialism to markets, depends on the kind of types that were observation equivalent in the absence of incentives. Furthermore, a firm has to attract the most suitable types for the firm's particular needs, which can be highly different across firms, e.g., between IT and public administration.

This last implication, incentives (possibly steep ones) for one category of types and tasks but no incentives (i.e., fixed wages) for another category allows for a (partial) explanation of some of the recently heavily debated differentiation and segmentation of the labor markets and the related increase of the Gini-coefficients in industrialized societies contrary to Kuznet's hypothesis and to trends during the whole 20th century. According to our findings, this can be partially attributed to the broad replacement of flat wages and hierarchies by incentives during the last decades. The introduction of incentives ranges from the CEOs down to the shop floor, from Western societies to former Communist or Socialist countries many of them developing countries. However, this change affected different industries and tasks differently, in particular, more in IT-related industries and services compared to old industries like steel manufacturing and to public administration, teaching etc. While there is no difference for fixed wages, dramatic differences emerge under incentives depending on which side-work ethic (or productivity) or intrinsic motive - a particular job falls. Intrinsic motives combined with multitasking seem to 
play a crucial role in professions like teaching, nursing, ${ }^{1}$ etc. Those jobs face already Baumol's cost disease and are in addition constrained in applying incentives.

For the example of climate change, Helm and Wirl (2016) already pointed out that the climate contracts in Martimort and Sand-Zantman $(2013,2016)$ as well as in Helm and Wirl (2014) differ substantially depending on whether private information is attached to the benefits or costs of mitigation efforts. To analyze this issue more systematically, in this paper we construct a model that is observation equivalent in the absence of contracts. Moreover, we explore the relevance of this issue in the widely applied setting of multi-tasking and outline some implications for the use of incentives in labor markets.

The chosen multitasking framework and its potential applications are related to many papers in industrial organization, labor and managerial economics. For example, Kragl and Schöttner (2014) consider the probability of completing a task by an agent engaged in two kinds of efforts with costs similar to our model, but no benefits from an intrinsic motivation. It falls into the line of multi-tasking papers going back at least to Feltham and Xie (1994), who analyze how to reward an agent on the basis of a performance measure, strictly separable cost functions, and risk. Baker (2002) emphasizes the trade-off between risk aversion and performance distortion in this framework. Schnedler (2008) allows for costs described by positive definite quadratic forms (as in our model) and emphasizes the signal to noise ratio associated with the performance measure.

\section{Model}

Our model builds on the multitasking framework in Benabou and Tirole (2016). The only modification is that the agents' productive efficiencies are replaced by their work ethic (i.e., disutility of effort), because this ensures 'observation-equivalence' with the second characterization of intrinsic motivation in the absence of monetary incentives.

An agent ('he') is engaged to provide two tasks: $x$ is observable but $y$ is not and can therefore not be incentivized. The principal ('she') earns the revenue $q$ from combining the agent's two tasks,

$$
q=a x+b y .
$$

The tasks require efforts $\left(e_{x}, e_{y}\right)$ that are costly for an agent,

$$
x=e_{x} \text { and } y=e_{y} .
$$

Therefore, the unobservable output $y$ is provided only due to an agent's intrinsic motive either the enjoyment or satisfaction from performing the $y$-task, or the feeling of an obligation 'to do what needs to be done'.

\footnotetext{
${ }^{1}$ E.g., Mullen et al. (2010) find no positive effect of pay for performance in the health sector.
} 
The principal's objective is to maximize the firm's total profit ${ }^{2}$ by aggregating over the set of agents $\Omega$ knowing the cumulative distribution function $F$,

$$
\max _{x \geq 0, w} \int_{\Omega}(q-w) d F,
$$

where $w$ denotes wage payments. The non-negativity of the observable output refers to the minimal (or reference) level that any agent must deliver in order to avoid being fired. Of course, the wage must be such that the agent accepts.

\section{Solution in the absence of incentive contracts}

\subsection{Work ethic $(t)$ is private information}

Type $t$ is the agent's private information that describes an agent's disutility of effort instead of his productive efficiency (or talent) as in Benabou and Tirole (2016). Effort costs are quadratic and include an interaction term with weight $\beta>0$, see (3). ${ }^{3}$ From the principal's perspective, $t \in[\underline{t}, \bar{t}]$ is a continuously distributed random variable characterizing an agent. We label the characteristic $t$ as work ethic although the agent of this type is intrinsically motivated too (the term $y$ in (3)) but this motivation is known and normalized since his payoff is,

$$
V=y-\frac{\alpha e_{x}^{2}+2 \beta e_{x} e_{y}+\gamma e_{y}^{2}}{2 t},
$$

in the absence of a wage. Accordingly, higher types $t$ face lower effort costs and are thus more efficient from the principal's perspective. An agent's choice of the necessarily unincentivized task conditional on the type and the incentivized task is (using $x=e_{x} \geq 0$ and $y=e_{y} \geq 0$ ),

$$
y(t, x)=\frac{t-\beta x}{\gamma}=\arg \max _{y} V .
$$

Therefore, the agent's $(V)$ and the principal's ( $q=$ aggregate output) gross payoffs (i.e., absent the wage) are,

$$
V(x, t)=\frac{t^{2}-2 t \beta x-\left(\alpha \gamma-\beta^{2}\right) x^{2}}{2 \gamma t},
$$

\footnotetext{
2 Only the total output aggregate over tasks and agents is observable. Alternatively, one could assume that the revenue $q$ is observable but non-verifiable in order to retain the essence of the multitasking model. Gersbach (1998) compares contracts based on the separate tasks with one based on $q$. Recently, Chen et al. (2019) introduce the concept of forcing contracts that make use of all information including the aggregate one.

3 Moving this interaction from the costs (3) to the revenues (1) eliminates multi-tasking and, as a consequence, also the problem we are interested in.
} 


$$
q(x, t)=a x+b \frac{t-\beta x}{\gamma}
$$

Remark 1 The choice of the characteristic work ethic instead of the agent's productivity as private information parameter has no effect in the case of a single output. Considering work ethic, a single and observable task $x$, the agent's payoff is,

$$
v(x, t)=-\alpha \frac{x^{2}}{2 t}
$$

after setting $y=e_{y}=0$ and using $x=e_{x}$ in the above quadratic costs of efforts (3).

Now assume that the types differ in their productivity, $x=e_{x} \sqrt{t}$, but are identical in their work ethic, $V=-\alpha \frac{e_{x}^{2}}{2}$, then the same gross payoff (7) results and consequently, the same incentives. Multi-tasking destroys the property of identical outcomes under work ethic and productivity. Indeed, the first point of the paper, different contracts for observation-equivalent characterizations, can be obtained for a single task and the characterizations productivity and intrinsic motivation (details are available upon request).

Remark 2 The chosen functional forms are not essential, because the whole analysis can be carried out with general specifications, e.g., $q=F(x, y)$, $V=u(y)-\frac{C(x, y)}{t}, F, u$ and $C$ meeting the standard assumptions.

\subsection{Intrinsic motive $(\theta)$ is private information}

An alternative description is that the level of the intrinsic benefit,

$$
\theta \in[\underline{\theta}=\underline{t}, \bar{\theta}=\bar{t}]
$$

characterizes an agent's type, while the work ethic is the same for all; we will use without loss in generality the normalization $E[t]=1=E[\theta]$. This yields for an agent's payoff,

$$
W=\theta y-\frac{1}{2}\left(\alpha e_{x}^{2}+2 \beta e_{x} e_{y}+\gamma e_{y}^{2}\right) .
$$

Maximization of $W$ with respect to the agent's choice of the unobserved task $y$ (using $x=e_{x}$ and $y=e_{y}$ ) yields

$$
y(\theta, x)=\frac{\theta-\beta x}{\gamma},
$$

which is identical to (4). Substituting (9) into (8), the agent's payoff becomes

$$
W(x, \theta)=\frac{\theta^{2}-2 \theta \beta x-\left(\alpha \gamma-\beta^{2}\right) x^{2}}{2 \gamma} .
$$

It is hard to reason a priori whether (5) or (10) characterizes the agent. In fact, both 
descriptions are observation-equivalent in the sense that the same actions result for no incentives.

Proposition 3 In the absence of monetary incentives (i.e., all types receive a fixed wage $\omega)$, the characterization of the agent's payoff according to (10) yields the same actions as (5),

$$
x_{0}(\theta)=x_{0}(t)=0, y_{0}(\theta)=\frac{\theta}{\gamma}=y_{0}(t)=\frac{t}{\gamma} .
$$

Therefore, the principal's payoff $q$ is also the same, (6) since $t=\theta$, and she prefers high types for both characterizations of the agent.

Proof Without an incentive contract (subscript 0), an agent characterized by his work ethic type $t$ chooses the following actions,

$$
x_{0}(t)=0=\underset{x \geq 0}{\arg \max } V \Longrightarrow y_{0}(t)=\frac{t}{\gamma} .
$$

The boundary solution $x_{0}=0$ results because $V_{x}<0$ at $x=0$ so that the agent's unconstrained choice of the observable output were negative. The constraint on the observable task (normalized to 0 ) forces the agent to deliver a minimum in order to receive the fixed wage $\omega$.

The same actions result for the types characterized by their intrinsic motive since

$$
x_{0}(\theta)=0=\underset{x \geq 0}{\arg \max } W \Longrightarrow y_{0}(\theta)=\frac{\theta}{\gamma} .
$$

and $\theta=t$. Given the same actions and $\theta=t$, the same payoff $q$ from (6) results for the principal. QED.

\section{Optimal incentive contracts}

\subsection{Assumptions}

We make the following assumptions for deriving the optimal incentive contracts for the above two alternative but observation equivalent characterizations of an agent's private information.

\section{Assumptions:}

1. The direct effects dominate, i.e., $\alpha \gamma>\beta^{2}$.

2. The joint payoff of principal and agent is increasing in the observable task, i.e., $q_{x}+V_{x}>0$ at least at low levels which is equivalent to,

$$
a>\frac{(1+b) \beta}{\gamma} \text {. }
$$

3. The types $t$ (and thus also $\theta$ ) are continuously (Benabou and Tirole 2016 consider a high and a low type) and uniformly distributed with $E[t]=1$, 


$$
f(t):=\frac{d F}{d t}=\frac{1}{\bar{t}-\underline{t}}=\frac{1}{2 \Delta}, t \in[\underline{t}:=1-\Delta, \bar{t}:=1+\Delta] .
$$

4. Since the restriction to participating types is not crucial (but other constraints play a crucial role), it is assumed that the agents' reservation utility $\underline{U}$ is sufficiently low such that the principal's wage offer attracts all types $t$ (see Remark 4 below). This is assumed for the $\theta$-types too.

5. Moving from a fixed wage $\omega$ to an incentive wage $w$ allows for two different cases (as in Benabou and Tirole 2016):(a) The principal's incentive contract must guarantee the agent a utility $U$ that is at least as large as the constant and exogenously given reservation utility $(R)$,

$$
R(t)=\underline{U} \text { and } R(\theta)=\underline{U},
$$

for all types. This scenario allows the principal to accrue parts of outside option (13) that an agent could earn under a fixed wage. (b) Since the fixed wage $\omega$ induces already type dependent payoffs to the agents,

$$
R(t)=\frac{t}{2 \gamma}+\omega, R(\theta):=\frac{\theta^{2}}{2 \gamma}+\omega,
$$

the offer of an incentive scheme must exceed those type dependent reservation utilities $R($.$) in order that the agent accepts the (new) incentive instead of the$ fixed wage. 4

Remark 4 The usual normalization, $\underline{U}=0$, is not suitable because it would allow for negative wages due to the agent's benefit from carrying out the intrinsically motivated task $y$. In particular, if $\underline{U} \leq \frac{t}{2 \gamma}$, all types will participate for any nonnegative wage. Therefore, $\underline{U}>0$ and also sufficiently large is necessary for a positive wage. If (and only if) the exogenous reservation utility $\underline{U}$ is sufficiently large, the principal can restrict the set of participating agents through her (constant) wage offer,

$$
\max _{\omega} \frac{1}{2 \Delta} \int_{2 \gamma(\underline{U}-w)}^{1+\Delta}\left(\frac{b t}{\gamma}-\omega\right) d t .
$$

That is, the principal can choose between a high wage $\omega$ and many, possibly all, types participating or a lower wage with a reduced set of participants delivering less aggregate output. A similar argument applies to $\theta$. However, by Assumption 4, it pays off for the principal to hire all types for which the inequality,

\footnotetext{
4 This is the case in many firms. See e.g., the case study in Lazear (2000) in which workers could but need not switch to the new incentive contract.
} 


$$
1-\Delta \geq \frac{2 \gamma \underline{U}}{1+2 b} \Longleftrightarrow \underline{U} \leq \frac{(1-\Delta)(1+2 b)}{2 \gamma}
$$

is satisfied.

Remark 5 Another interpretation of the type dependent reservation utility in Assumption $5 \mathrm{~b}$ is that the agent's payoff results from self-employment. Then it makes sense to assume that higher types (whether intrinsically motivated or with a higher work ethic) have a better outside option.

\subsection{Work ethic}

The revelation principle allows to restrict the analysis to truth telling incentives, i.e.,

$$
U(t):=U(t, t) \geq U(\hat{t}, t):=V(x(\hat{t}), t)+w(\hat{t}) \forall t, \hat{t} \in[\underline{t}, \hat{t}],
$$

where $U(\hat{t}, t)$ denotes the payoff of type $t$ pretending to be type $\hat{t}$. The second constraint is that only agents receiving at least their reservation utility $(R)$ will accept the offer.

Using $U(t)$ defined in (15), the principal has to solve the following optimal control problem (suppressing the function argument $t$ ),

$$
\begin{gathered}
\max _{x \geq 0} \int_{\underline{t}}^{\bar{t}}\left(\frac{b t+(a \gamma-b \beta) x}{\gamma}+\frac{(t-2 \beta x) t-\left(\alpha \gamma-\beta^{2}\right) x^{2}}{2 \gamma t}-U\right) d F, \\
\dot{U}=V_{t}=\frac{t^{2}+\left(\alpha \gamma-\beta^{2}\right) x^{2}}{2 \gamma t^{2}}, \\
U \geq R \forall t \in[\underline{t}, \bar{t}] .
\end{gathered}
$$

Of course, only the observable output $x$ can serve as an instrument for incentives. The objective (16) results from solving (15) at $\hat{t}=t$ for the wage $w(t)$, substituting this into the principal's payoff (6), rearranging and integrating over all types. The differential equation (17) is the so-called incentive compatibility constraint that is implied by the first order condition that truth telling must be an agent's dominant strategy (this condition is here also sufficient). Inequality (18) is the participation constraint: an agent will accept the incentive contract only if the state inequality constraint (18) holds, i.e., his payoff exceeds his reservation utility $R$, which can be constant (Assumption 5a) or type dependent (Assumption 5b). A solution of the above optimal control problem (16)-(18) is implementable if it is monotonic in the prescription of tasks, i.e., $\dot{x} \geq 0$.

Proposition 6 The optimal prescription of the observable task along an interior contract follows the 'relaxed program' identified by the subscript $r$, 


$$
x_{r}(t)=\frac{(a \gamma-\beta(b+1)) t^{2}}{\left(\alpha \gamma-\beta^{2}\right) \bar{t}}>0,
$$

which satisfies the condition of monotonicity, $\dot{x}_{r}>0$. Considering the unobservable task, the agent delivers

$$
y_{r}(t)=\frac{t}{\gamma}\left(1+\frac{\beta(\beta(b+1)-a \gamma) t}{\left(\alpha \gamma-\beta^{2}\right) \bar{t}}\right) .
$$

Proof The assumption that the direct effects dominate, i.e. $\alpha \gamma>\beta^{2}$, implies that $\dot{U}>0$. Therefore, the participation constraint binds only at the lowest type $t=\underline{t}$ irrespective of our assumptions about the reservation utility: trivially for the constant reservation utility but also for the type dependent and increasing reservation utility $R(t)$ because

$$
\dot{U}-\dot{R}=\frac{\left(\alpha \gamma-\beta^{2}\right) x^{2}}{2 \gamma t^{2}}>0 .
$$

Therefore, the solution follows from the relaxed program condition that the marginal increase in the total surplus must equal the expected marginal increase in the agent's rent (see e.g., Fudenberg and Tirole 1992, p. 265), i.e. from

$$
q_{x}+V_{x}=\frac{V_{x t}}{h},
$$

where $h:=f /(1-F)$ denotes the hazard rate and $1 / h=\bar{t}-t$ for the uniform distribution; $q_{x}, V_{x}$ and $V_{t x}$ follow from differentiating (5) and (6). Upon substitution,

$$
a-\frac{\beta(b+1)}{\gamma}-\frac{\left(\alpha \gamma-\beta^{2}\right) x}{\gamma t}=(\bar{t}-t) \frac{\left(\alpha \gamma-\beta^{2}\right) x}{\gamma t^{2}},
$$

and solving (22) for $x$ yields (19). Assumption 2 ensures $x_{r}>0$ and monotonicity, $\dot{x}_{r}>0$. The unobservable output (20) follows from substituting (19) into (4).

From Assumption 2, the ratio between the brackets in (20) is negative. Therefore, $y_{r}<0$ can result for large tasks $x_{r}$ and the principal cannot deter this negative output directly. This lack of observability of $y$ introduces an asymmetry compared to the verifiable output $x$ for which the principal can ensure non-negativity. This possibility of $y<0$ is a consequence of the assumed quadratic specification (5) in which negative efforts can lower the agent's cost. This need not be implausible, e.g., the agent may enjoy sabotaging the unobservable task $y$ if it mitigates his disutility from delivering a large $x .^{5}$ Indeed, if $a$ is sufficiently large, the principal may prefer

\footnotetext{
5 For example, $x$ denotes the output using an equipment and $y$ the care exercised with the use of this (expensive) equipment. Asking for high levels of $x$ will not only reduce care but an angry agent may ignore certain warnings or even kick the machine.
} 
this sabotage as long as it helps to increase the much more important $x$. Alternatively, she can avoid $y<0$ (or even if necessary ensure $y \geq y^{\min }>0$ ) by constraining her demands on the observable output. This is addressed in Sect. 6.

Not only tasks but also the wages are the same for both assumptions about the agent's reservation utility, $5 \mathrm{a}$ or $5 \mathrm{~b}$, because setting,

$$
R(\underline{t})=\underline{U}=\frac{\underline{t}}{2 \gamma}+\omega,
$$

implies $U(t)>R(t)=\frac{t}{2 \gamma}+\omega>\underline{U}$ for all $t>\underline{t}$ so that the participation constraint binds in both cases only for $t$ and the agents' payoffs $U$ are the same. The wage follows in both cases from (15) after integrating (17),

$$
\begin{gathered}
w(t)=\int_{\underline{t}}^{t} \frac{z^{2}+\left(\alpha \gamma-\beta^{2}\right) x_{r}^{2}(z)}{2 \gamma z^{2}} d z+R(\underline{t})-V\left(x_{r}(t), t\right) \\
=\frac{(a \gamma-\beta(b+1))}{2 \gamma}\left(\frac{(a \gamma-\beta(b+1))\left(4 t^{3}-\underline{t}^{3}\right)+6 \beta t^{2} \bar{t}}{3\left(\alpha \gamma-\beta^{2}\right) \bar{t}^{2}}\right)+\underline{U},
\end{gathered}
$$

if possibly binding constraints accounting for $y \geq 0$ are ignored.

Proposition 7 Which assumption about the reservation utility (5a or 5b) constrains the implementation of incentives has no effect, neither on tasks nor on wages.

Figure 2 shows an example of the corresponding contract in comparison with the alternative derived in the next subsection. It exhibits the familiar features: observable output and wage increase with an agent's work ethic. The output of the unobservable task is substantially less than in the absence of monetary incentives and in this example its dependence on types is weak and non-monotonic, because

$$
\dot{y} \geq 0 \Longleftrightarrow \frac{2 \beta(\beta(b+1)-a \gamma)}{\alpha \gamma-\beta^{2}} \geq-\frac{\bar{t}}{t} .
$$

\subsection{Intrinsic motivation}

We now turn to the alternative specification and treat the intrinsic motive $\theta$ as private information while the work ethic is known, $t=E[t]=1$. The analysis proceeds as above. By the revelation principle, the payoff of an agent who reveals his type must satisfy (in slight abuse of notation, we use the same letter $U$ in spite off a different argument $\theta$ instead of $t$ in (15) and, as we will see, a different payoff),

$$
U(\theta):=W(\theta, \theta)+w(\theta) \geq W(\hat{\theta}, \theta)+w(\hat{\theta}) \forall \hat{\theta}, \theta \in[\underline{t}, \vec{t}],
$$

where (using 10) 


$$
W(\hat{\theta}, \theta)=\frac{\theta^{2}-2 \theta \beta x(\hat{\theta})-\left(\alpha \gamma-\beta^{2}\right) x^{2}(\hat{\theta})}{2 \gamma} .
$$

Now treating the intrinsic motive $\theta$ as private information and the work ethic as known yields the following optimal control problem,

$$
\begin{gathered}
\max _{x \geq 0} \int_{\underline{\theta}}^{\bar{\theta}}\left(\frac{b \theta+(a \gamma-b \beta) x}{\gamma}+\frac{(\theta-2 \beta x) \theta-\left(\alpha \gamma-\beta^{2}\right) x^{2}}{2 \gamma}-U\right) d F, \\
\dot{U}=W_{\theta}=\frac{\theta-\beta x}{\gamma}, \\
U \geq R \forall \theta \in[\underline{\theta}, \bar{\theta}] .
\end{gathered}
$$

The objective (28) is very similar to (16) and identical concerning the principal's gross payoff (the two ratios under the two integrals). The interpretation of the constraints (29) and (30) is as in the case of work ethic.

Although the multitasking problem looks in this form similar to the one above and is observation equivalent in the absence of incentives due to Proposition 3 , there are noteworthy differences (1) between the objectives ( 16) versus (28), (2) the incentive compatibility constraints (17) versus (29) and (3) the participation constraints and here only under Assumption $5 \mathrm{~b}$ due to differences in $R$ according to (13). Most importantly, a substantially different mechanism results.

\subsubsection{Type dependent reservation utility}

We consider first the scenario $5 \mathrm{~b}$ of type dependent agent reservation utility. This leads to a reversal of the ordering of the agents' efficiencies, because

$$
\dot{U}-\dot{R}=\frac{\theta-\beta x}{\gamma}-\frac{\theta}{\gamma}=-\frac{\beta x}{\gamma}<0,
$$

although $\dot{U}=W_{\theta}=y$ can be positive and is positive iff $y>0$. Tying an incentive scheme to the agent's reservation utility on the left hand side, $U=R$ (at $\theta=\underline{\theta}$ any other marginal type as is expected from $\dot{U}>0$ ), implies $U<R$ for all larger types and thus violates the participation constraint (30). Hence, the marginal type must be on the right hand side and moreover at the top $\bar{\theta}$. This type receives his reservation utility, here $R(\bar{\theta})$. The lower types $\theta<\bar{\theta}$ earn payoffs higher than for the fixed wage, $U(\theta)>R(\theta)$ satisfying the participation constraint due to $\dot{U}=y<y_{0}$ for any $x>x_{0}=0$. The lowest type $\underline{\theta}$ produces the first best (no distortion from the 'top') and is thus the most efficient one from the principal's perspective.

Proposition 8 Focusing on interior non-negative tasks and the type dependent reservation utility from (13), the principal prescribes 


$$
x_{r}(\theta)=\frac{a \gamma+\beta(\underline{\theta}-b)-2 \beta \theta}{\alpha \gamma-\beta^{2}} .
$$

The tasks $x_{r}(\theta)$ are linearly declining in $\theta$ if

$$
a>\frac{\beta(b+2 \bar{\theta}-\underline{\theta})}{\gamma},
$$

and thus reach the maximum at $\underline{\theta}$ (identical to the first best). This contract induces the agent to deliver

$$
y_{r}(\theta)=\frac{\theta}{\gamma}-\frac{a \gamma+\beta(\underline{\theta}-b)-2 \beta \theta}{\left(\alpha \gamma-\beta^{2}\right) \gamma}
$$

of the unobserved output, which increases linearly with respect to $\theta$.

Proof The derivation is similar to Proposition 6. The relaxed program follows from the condition (the hazard rate is now different for a mechanism in which the most efficient type is at the bottom),

$$
q_{x}+W_{x}=-\frac{F}{f} W_{x \theta},
$$

thus

$$
a-\frac{\beta(b+\theta)}{\gamma}-\frac{\left(\alpha \gamma-\beta^{2}\right) x}{\gamma}=\frac{(\theta-\underline{\theta}) \beta}{\gamma}
$$

so that (32) results. This program satisfies the monotonicity constraint, here $\dot{x}_{r}<0$, and it is optimal to offer this interior contract to all types as long as $x_{r}>0$, which is ensured for the slightly sharpened version of Assumption 2 in (33).

The tasks in (32) and (34) are backed up by the wage that follows this time from backward integration:

$$
w_{r}(\theta)=\int_{\bar{\theta}}^{\theta} \frac{z-\beta x_{r}(z)}{\gamma} d z+R(\bar{\theta})-W\left(x_{r}(\theta), \theta\right)
$$

and after substituting $R(\bar{\theta})$ from (13) according to Assumption 5b,

$$
w_{r}(\theta)=\frac{(a \gamma-b \beta)^{2}+2 \beta(\underline{\theta}+\bar{\theta}-2 \theta)(a \gamma-b \beta)+\beta^{2}\left((\underline{\theta}+\bar{\theta}-2 \theta)^{2}-2(\bar{\theta}-\theta)^{2}-\bar{\theta}^{2}\right)}{2 \gamma\left(\alpha \gamma-\beta^{2}\right)} .
$$

The principal prefers high types if not offering incentives $\left(x_{0}\right.$ and $y_{0}$ from Proposition 3 ) and also for incentivizing the $t$-types (the point of no distortion is at the top at $\bar{t}$ ). Moreover $\theta=t$. Nevertheless, low types $\theta$ are more efficient if incentivized according to Proposition 8 and the point of no distortion (and thus the largest task $x$ ) 
is at the bottom at $\underline{\theta}$. This upside-down ordering of agents' efficiencies after introducing incentives and compared with the observation equivalent characterization in terms of work ethic is puzzling. Economically, a large $\theta$ characterizes an agent with a high intrinsic motive of providing the necessarily unincentivized task $(y)$, which makes it more costly to move him to the observable task $(x)$, which is more important for the principal by assumption 3. Furthermore, a high $\theta$ improves the agent's outside option, which makes contracts more expensive for the principal. Figure 2 highlights the properties of this contract for a particular example and the difference to contracting with agents having private information about their work ethic.

\subsubsection{Constant reservation utility}

The alternative assumption 5a, $U(\theta) \geq \underline{U}=$ constant, affects only the IR constraint (30) and has no consequences in the case of work ethic. However, matters are again different for intrinsically motivated agents and this modest change of one assumption has far fetching consequences beyond the already addressed reversal of the efficiency of types. More precisely, although Assumption 5a does not affect the relaxed program (32) and its proper monotonicity, $\dot{x}<0$, it rules out the implementation of the relaxed program. The reason is that the agent's payoff $U$ must decline if the participation constraint binds at $\bar{\theta}$. However,

$$
\dot{U}<0 \Longleftrightarrow \frac{\theta-\beta x}{\gamma}<0 \Longleftrightarrow y<0 .
$$

Therefore, a corresponding interior contract is only implementable for $y<0$, i.e., if the principal accepted negative, i.e., damaging performance on the unobservable task, at least for some types.

Not even more complex contracts are possible, neither countervailing ones, which consist of the boundary solution connecting left-right and right-left mechanism (Maggi and Rodriguez-Clare 1995) nor ones with no distortion in the interior (Kerschbamer and Maderner 1998; 2000; Wirl and Huber 2005).

Proposition 9 Only fixed wage contracts are possible if the agents have a constant and exogenously fixed reservation utility $\underline{U}$ and the principal wants to ensure $y \geq 0$. The optimal fixed wage contract results from 'bunching', i.e., all types are asked to produce the first best of the observable task of the lowest type (no distortion at the bottom),

$$
x(\theta)=x^{1}(\underline{\theta})=\frac{a \gamma-b \beta-\beta \underline{\theta}}{\alpha \gamma-\beta^{2}},
$$

which nevertheless induces type dependent outputs of the unobservable task,

$$
y(\theta)=\frac{\theta}{\gamma}-\frac{\beta}{\gamma} \frac{\alpha \gamma-b \beta-\beta \underline{\theta}}{\alpha \gamma-\beta^{2}},
$$

if $y \geq 0$. The principal offers the fixed wage, 


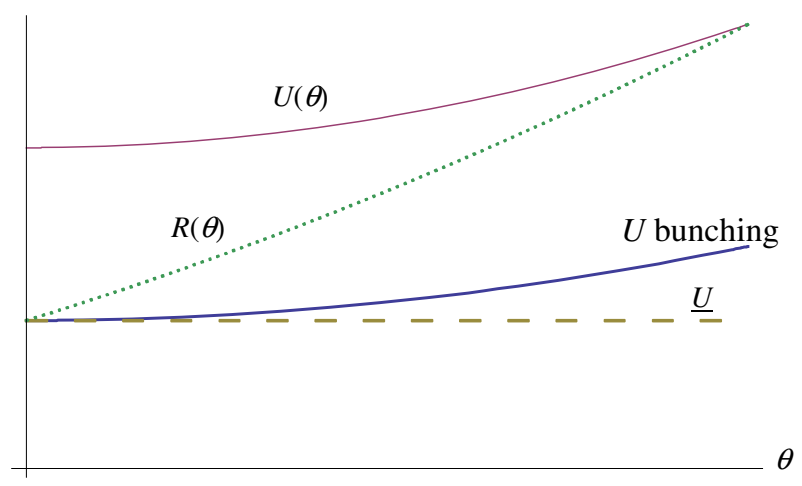

Fig. 1 Comparing payoffs of agents with respect to their intrinsic motive (parameterized by $\theta$ )

$$
\omega^{*}=\underline{U}-W\left(x^{1}(\underline{\theta}), \underline{\theta}\right)
$$

to all types, which ensures the participation of the lowest type (just) and thus of all others, who earn an information rent, $U>\underline{U}$.

Figure 1 (although qualitative, it is based on the example introduced in the next section) plots the agents' payoffs across the different scenarios: $\underline{U}$ is the agent's constant outside option; $R(\theta)$ is the net rent that the agent earns in the absence of incentives and for the fixed wage $\omega$ from Proposition 3, i.e., $R(\theta)$ from (13); $U(\theta)$ is the agent's payoff if the principal is bound by the type dependent reservation utility $R(\theta)$ and implements the contract from Proposition 8; finally $U$ is the (also type dependent) payoff if the principal applies the bunching strategy from Proposition 9.

\section{Comparison}

There are stark differences between the two characterizations despite observation equivalence. Work ethic allows for interior contracts with nonnegative outputs (the analysis of the constraint $y \geq 0$ is the subject of the next section) irrespective of the details of the participation constraint. In contrast, intrinsic motivation does not allow for incentives at all, if the reservation utility is constant and negative outputs of the unobservable task must be avoided. Under the hypothesis of a type dependent reservation utility, an optimal contract exists in which the characterization of the efficient type is turned upside-down compared with the starting point of no incentives and the case of work ethic.

Figure 2 compares the contracts in their direct form, i.e. as functions of the agents' types, $t$ or respectively $\theta$, assuming that the observable output is twice as important as the unobservable one, that both kinds of efforts are equally costly and lead to significant cost spillovers $(\beta=1 / 2)$, that the types vary up to $25 \%$ from the 

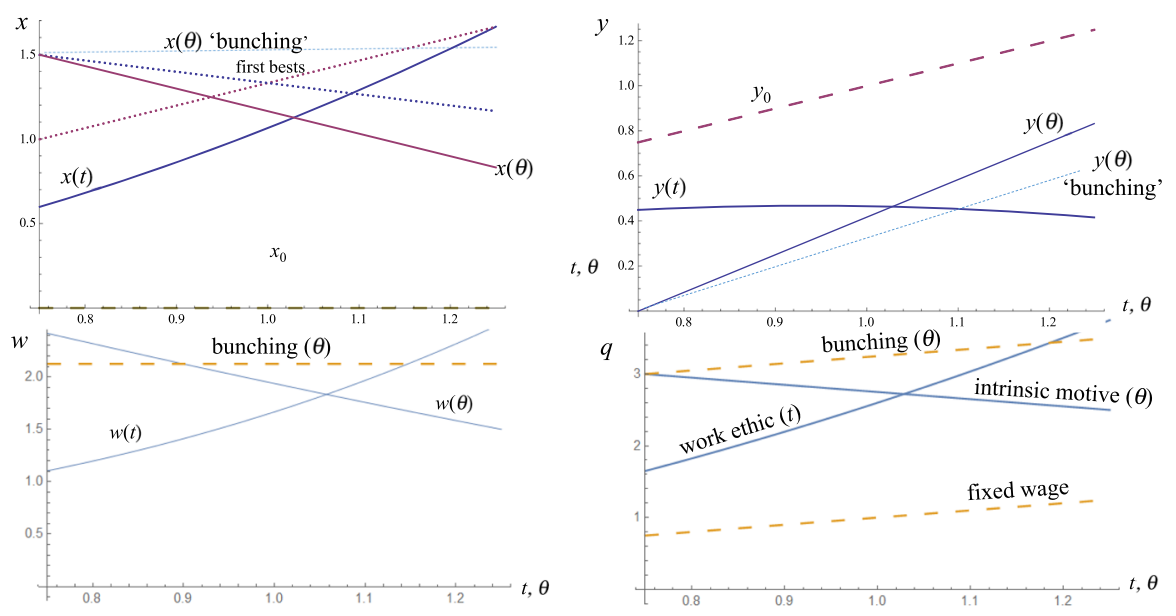

Fig. 2 Comparing optimal incentives ( $t=$ work ethic, $\theta=$ intrinsic motive to produce $y)$, $a=2, b=1, \underline{t}=3 / 4, \bar{t}=5 / 4, \alpha=1, \beta=1 / 2, \gamma=1, \underline{U}=1$

mean, and that the reservation utility is chosen such that the necessary wage is positive in spite of the intrinsic benefit ${ }^{6}$, i.e., for the parameter values:

$$
a=2, b=1, \alpha=1, \beta=1 / 2, \gamma=1, \underline{t}=\underline{\theta}=3 / 4, \bar{t}=\bar{\theta}=5 / 4, \underline{U}=1 .
$$

The comparison focuses on the Assumption 5b, i.e., the principal's offer must exceed an agent's payoff working for a fixed wage for the intrinsic motive i.e., $U \geq R$ from (13). Figure 2 highlights how assignments ( $x$ and implicitly $y$ ), wages $(w)$, payoffs for the principal $(q)$, and the locations of the efficient type (at $\bar{t}$, and respectively, at $\underline{\theta}$ ) differ depending on whether the agent is described either by his work ethic $(t)$ or his intrinsic motive $(\theta)$. The differences in this example suggest: First, the observable output of the average types, $t=\theta=1$, is larger if the agent is characterized by his intrinsic motive. Second, incentives tied to work ethic induce a larger variation in the observable activity. And as a corollary, the opposite holds for the unobservable task that varies much more if agents are characterized by their intrinsic motive due to (4) and (9). While the second observation is in line with intuition, the first is surprising, because it is the average of the intrinsically motivated type that allows for higher output of the observable task. However, do these properties hold generally, at least along interior mechanisms (i.e., the relaxed programs)? Both hold but with additional qualifications that the observable output is of sufficient importance, more than what is assumed in Assumption 2, see Appendix. A consequence of less variation in $x_{r}(\theta)$ is a wider variation in $y_{r}(\theta)$ such that the constraint $y \geq 0$ is more likely to bind for $\theta$-types. Indeed, this constraint is just avoided in the example in Fig. 2 for the $\theta$-types, $y_{r}(\underline{\theta})=0$, while $y_{r}(t)>0 \forall t$.

The chart on the bottom, left hand side of Fig. 2 shows that the highest wages go to the type with the highest work ethic $(\vec{t})$ but to the type with the least intrinsic

\footnotetext{
6 The agent's payoffs under a fixed wage are (13). The usual assumption $\underline{U}=0$ allowed for negative wages which $\underline{U}=1$ rules out for the parameters below.
} 


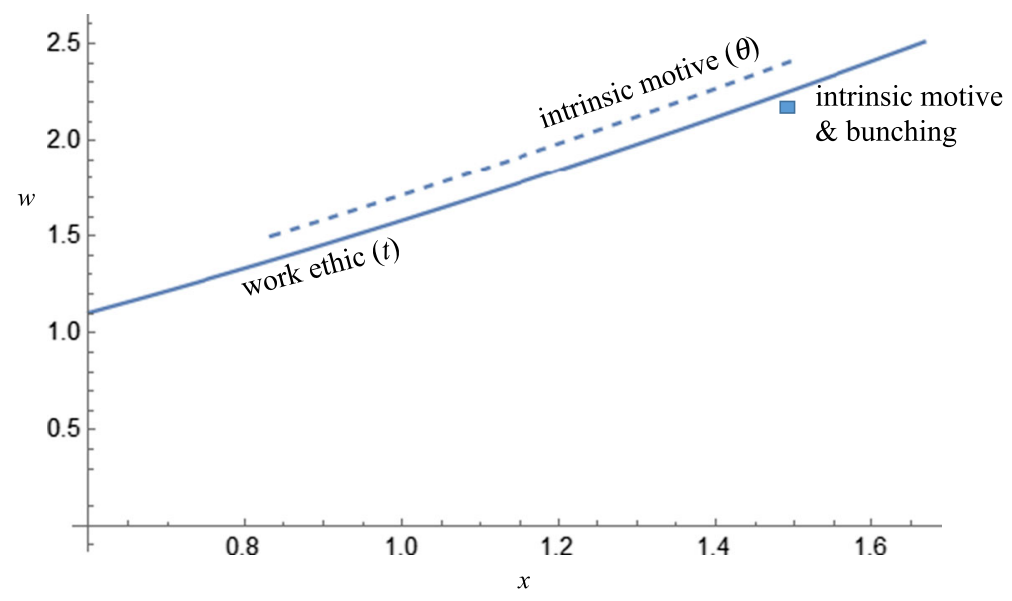

Fig. 3 Comparing optimal incentive contracts: wages contingent on the observable task, $a=2, b=1, \underline{t}=3 / 4, \bar{t}=5 / 4, \alpha=1, \beta=1 / 2, \gamma=1, \underline{U}=1$

motivation $(\underline{\theta})$. The wages show a wider variation across the $t$-types because they must be compensated for a larger variation in $x$. However, the large differences disappear if the wage $w$ is plotted versus the observable output $x$, see Fig. 3 .

The Assumption 5a of an exogenously fixed and type independent reservation utility has no effect on the tasks in the case of work ethic as an agent's characteristic. However, Assumption 5a affects the intrinsically motivated type: it leads to a constant demand for the observable task backed up by a constant wage, the "bunching" (see Fudenberg and Tirole 1992 on this terminology) policy from Proposition 9. Therefore, the contract is substantially different from the one derived under Assumption 5b. Fig. 2 highlights that Assumption 5a allows the principal to obtain a significantly higher output (in terms of $x$ as well as aggregated) from her workers and this rather than the ability to pay a lower wage explains why this policy is more profitable.

\section{Non-negativity constraints}

One conclusion from the above analysis seems that the assumption about the agent's private information (either $t$ or $\theta$ ) matters but not too much (at least under Assumption 5b), because both imply a similar output-wage relationship in spite of the disagreement which types are efficient, see Fig. 3. Therefore it seems that the principal does not need to care much whether her agents are intrinsically motivated or have a good work ethic. However, what about the consequences of binding nonnegativity constraints? First, the relaxed programs of the observable output can turn negative, which is assumed away in Assumption 2. Yet if such outcomes existed for 
types along the relaxed program (i.e., $t: x_{r}(t)<0$ and $\theta: x_{r}(\theta)<0$ ), ${ }^{7}$ then it would be easy to handle this constraint $x \geq 0$ : a fixed wage leading to the non-negative actions $x_{0}=0$ and $y_{0}>0$ from Proposition 6 in both cases. This is incentive compatible because it allows for a continuous and monotonic joining in efforts and payoffs (in the case of intrinsic motives only under Assumption 5b).

Second, the unobservable task can turn negative along the relaxed program, $y_{r}<0$, which follows from the explicit solutions. The example in Fig. 2 avoids this only just for the $\theta$-types, which are more likely to face this constraint. By its very nature, non-negativity of the unobservable tasks cannot be directly ensured. However, given the importance of the unobservable task, the principal can constrain her demands on $x$ in order to ensure that $y$ surpasses a crucial threshold (normalized to 0 ), by adding the control constraints to the optimization problems,

$$
\begin{aligned}
& x(t) \leq \frac{t}{\beta}, \\
& x(\theta) \leq \frac{\theta}{\beta} .
\end{aligned}
$$

Those constraints are identical for both characterizations and, if at all, can only affect types who are asked to produce a large observable output along the relaxed program. Therefore, accounting for this implicit non-negativity constraint on the unobservable output weakens the power of incentives over and above the one resulting from multitasking.

In spite of their arithmetical identity, the two control constraints have strongly different implications on incentives. The constraint (41) for the $t$-types poses no problem, because it does not affect the ordering: a higher type is asked to produce more of the observable output whether in the interior along $x_{r}(t)$ or along the constraint. Therefore, joining of output, effort and wages between the unconstrained $\left(x=x_{r}<t / \beta\right)$ and the constrained $(x=t / \beta)$ domain remains continuous, and satisfies individual rationality, incentive compatibility and monotonicity for all types. However, things are different for the $\theta$-types, because the incentivized task, $x=x_{r}$ from (32), declines with respect to $\theta$ yet must increase with respect to $\theta$ along the constraint (42). Combining (32) and (42) violates monotonicity and implies that identical levels of $x$ are assigned to two different types which cannot be incentive compatible.

Proposition 10 If the range of types (i.e., the parameter $\Delta$ ) exceeds a threshold,

$$
\Delta>1-\frac{(a \gamma-b \beta) \beta}{\alpha \gamma},
$$

or respectively the observable output is sufficiently important (i.e., a is sufficiently large), then it is impossible to offer incentives and to meet the non-negativity constraint on the unobservable task for the $\theta$-types

\footnotetext{
${ }^{7}$ Although unlikely, negative outcomes are at least theoretically conceivable. For example, Google does not fire all of those programmers, who deliver less than the required lines of code (= our reference level $x=0)$ in the hope that they think more about the next killer app.
} 
Proof Denote with $\tilde{\theta}$ the type at which $y \geq 0$ becomes binding. Using the explicit solution (34) implies $\tilde{\theta} \in(\underline{\theta}, \bar{\theta}]$

$$
\tilde{\theta}=\frac{(a \gamma+(\underline{\theta}-b) \beta) \beta}{\alpha \gamma+\beta^{2}}>\underline{\theta}=1-\Delta \Longleftrightarrow \Delta>\frac{\alpha \gamma+b \beta^{2}-a \beta \gamma}{\alpha \gamma}
$$

and thus iff

$$
a \gamma-b \beta>(1-\Delta) \frac{\alpha \gamma}{\beta} \text {. QED. }
$$

Since, the reference example in Figs. 2 and 3 avoids this complexity entirely for the $t$-types but only just for the $\theta$-types, Fig. 4 shows an example in which $y \geq 0$ binds for both characterizations of types. This creates no problem for incentivizing $t$-types: the constraint ( 41) replaces the relaxed program at high types retaining monotonicity. Those then lower outputs are rewarded with a lower wage (due to smaller increases in $U$ and less compensation for lowered efforts). However, incentives are impossible if the principal wants to implement $y(\theta) \geq 0$ for agents with private information about their intrinsic motives, because joining the declining interior with the increasing boundary solution violates monotonicity. Hence, only fixed wages are implementable.

Summarizing, agents differing in their work ethic allow combining incentives with a mitigation of the multitasking problem by accounting for minimal requirements on the unobservable task. However, this does not need to hold for agents differing in their intrinsic motive. In this case, as in the example in Fig. 4, the principal will have to offer fixed wages. The reason is that the constraint imposed by multitasking (i.e., $y \geq 0$ or some other target on the unobservable task) rules this out

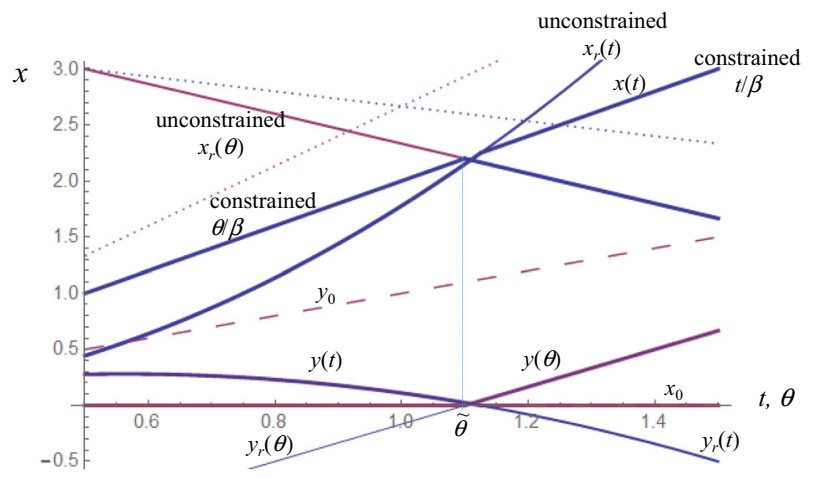

Fig. 4 Observable output depending on whether work ethic $(t)$ or intrinsic motive $(\theta)$ is the agent's private information when $\mathbf{y} \geq \mathbf{0}$ is binding, $a=3, b=1, \alpha=1,=1 / 2, \gamma=1$; dotted lines indicate the first bests 
in one case $(\theta)$ but not in the other $(t)$ although both descriptions are observationequivalent in the absence of incentives.

The results have important implications for managerial economics and labor markets: Agents, characterized by their work ethic can be incentivized in all circumstances, while the observation-equivalent characterization of intrinsic motivation can render incentives impossible, here for an exogenously fixed reservation utility and for binding constraints on the unobservable task. Therefore, managers should use incentives in the first case but much more cautiously or not at all in the second case. This is empirically testable. For example, monetary incentives should be less important in businesses where intrinsic motives are crucial and multitasking issues must be solved. Furthermore, these differences may induce firms to offer either incentives or fixed wages in order to attract the optimal type of agents.

\section{Concluding remarks}

The analysis started from different payoff formulations of a multitasking problem (a slight modification of the one used in Benabou and Tirole 2016) that are observational equivalent without an incentive contract. However, the incentive contracts implied by seemingly equivalent descriptions are different. Not only that, the very characterization which agent type is efficient is turned upside down for incentivizing intrinsically motivated agents. Furthermore the feasibility of incentivizing agents exposed to multiple tasks is severely restricted in one (intrinsic motive) but not in the other (work ethic) characterization of agents. Since multitasking is important for many real world labor relations, getting the incentives right is crucial. However, only one type (those characterized by their work ethic) can be incentivized but not another type (here those with intrinsic motives) although both types behave identical in the absence of incentives.

While we can explain why seemingly equivalent normalizations lead to different contracts, an additional explanation is necessary for the stark differences. The first point, the reversal of efficient types, is an implication of multitasking that arises also in the setting of Benabou and Tirole (2016), which is here strengthened by departing from observation equivalent setups. The second point is that one description (work ethic) allows for incentives but they can be impossible for agents described by an observation equivalent characteristic (here intrinsic motives). Embedding this lack of incentives contracts into the analysis of firms competing for either hard working ( $t$-types) or highly motivated ( $\theta$-types) agents strengthens the observation in Benabou and Tirole (2016) that (upward) wage distortions due to competition for motivated types are not only attenuated but may be even eliminated. Indeed, the same inexistence result can be established in the Benabou and Tirole (2016) framework for continuously distributed types; detail is available upon request. However, this last conclusion must be attributed to multitasking, because otherwise one can show that intrinsic motives lead to excessive wages, even over-paying stars, as can be observed in sports, business and arts, compare Wirl (2003). 
These observations lead to many questions that could be the subject of future research. First, to test empirically the implication that incentives are applied in the case of work ethic (or productive efficiency) but much less in environments relying on intrinsically motivated agents in settings of multitasking. Second, to check in analytical models with different applications whether the optimal mechanism is robust if a different (but possibly observation equivalent) private information parameter is chosen. Third, to apply our observation equivalent multitasking set ups to topical issues of labor markets (like its diversification along the lines just sketched above) similar to the application in Benabou and Tirole (2016) in order to investigate bonus culture. Fourth, to address specific aspects of industries such as the IT industry, analytically and/or empirically. Fifth, one may add risk aversion on the side of the agent. Last, one may embed the suggested multi-tasking framework into Mirrlees' (Mirrlees 1971) framework of optimal taxation in which the unobservable task (like caring about one's children and their education) has social value and how different agents' characteristics (work ethic, intrinsic motive for the unobservable task) call for different tax regimes.

Acknowledgements Open access funding provided by University of Vienna. I benifitted from discussions with Markus Reitzig about the specificics of IT developments. Both authors acknowledge helpful comments from an anonymous referee.

Open Access This article is licensed under a Creative Commons Attribution 4.0 International License, which permits use, sharing, adaptation, distribution and reproduction in any medium or format, as long as you give appropriate credit to the original author(s) and the source, provide a link to the Creative Commons licence, and indicate if changes were made. The images or other third party material in this article are included in the article's Creative Commons licence, unless indicated otherwise in a credit line to the material. If material is not included in the article's Creative Commons licence and your intended use is not permitted by statutory regulation or exceeds the permitted use, you will need to obtain permission directly from the copyright holder. To view a copy of this licence, visit http:// creativecommons.org/licenses/by/4.0/.

\section{Appendix for referees (online?)}

\section{Second order condition}

\section{Work ethic is private information}

$$
\begin{aligned}
U(t, \hat{t}) & =y(t, x(\hat{t}))-\frac{\alpha x^{2}(\hat{t})+2 \beta x(\hat{t}) y(t, x(\hat{t}))+\gamma y^{2}(t, x(\hat{t}))}{2 t}+w(\hat{t}) \\
& =\frac{(t-\beta x(\hat{t}))^{2}}{2 t \gamma}-\frac{\alpha x^{2}(\hat{t})}{2 t}+w(\hat{t})
\end{aligned}
$$

Therefore,

$$
U_{\hat{t}}=-\frac{(t-\beta x(\hat{t})) x(\hat{t}) \dot{x}(\hat{t})}{\gamma t}-\frac{\alpha x(\hat{t}) \dot{x}(\hat{t})}{t}+\dot{w}(\hat{t})
$$

and 


$$
U_{\hat{t} \hat{t}}=\ddot{w}(\hat{t})-\frac{\left(\alpha \gamma-\beta^{2}\right) \dot{x}^{2}(\hat{t})}{\gamma t}-\frac{\left(\beta t+\left(\alpha \gamma-\beta^{2}\right) x(\hat{t})\right) \ddot{x}(\hat{t})}{\gamma t} .
$$

From the revelation principle, $\hat{t}=t$ and substituting the derivatives from the relaxed program solution yields,

$$
U_{t t}=\frac{2((1+b)-a \gamma) x(t)}{\gamma t \bar{t}}<0
$$

which is negative by Assumption 2 .

\section{Intrinsic Motive is private information}

$$
\begin{aligned}
U(\hat{\theta}, \theta) & =\theta y(\theta, x(\hat{\theta}))-\frac{1}{2}\left(\alpha x^{2}(\hat{\theta})+2 \beta x(\hat{\theta}) y(\theta, x(\hat{\theta}))+\gamma y^{2}(\theta, x(\hat{\theta}))\right)+w(\hat{\theta}) \\
& =-\frac{\alpha}{2} x^{2}(\hat{\theta})+\frac{(\theta-\beta x(\hat{\theta}))^{2}}{2 \gamma}+w(\hat{\theta})
\end{aligned}
$$

Therefore,

$$
U_{\hat{\theta}}=-\frac{\left(\beta \theta+\left(\alpha \gamma-\beta^{2}\right) x(\hat{\theta})\right) \dot{x}(\hat{\theta})}{\gamma}+\dot{w}(\hat{\theta})
$$

and

$$
U_{\hat{\theta} \hat{\theta}}=\ddot{w}(\hat{\theta})-\frac{\left(\alpha \gamma-\beta^{2}\right) \dot{x}^{2}(\hat{\theta})}{\gamma}-\frac{\left(\beta \theta+\left(\alpha \gamma-\beta^{2}\right) x(\hat{\theta})\right) \ddot{x}(\hat{\theta})}{\gamma}
$$

since $\ddot{x}=0$,

$$
U_{\hat{\theta} \hat{\theta}}=\ddot{w}(\hat{\theta})-\frac{\left(\alpha \gamma-\beta^{2}\right) \dot{x}^{2}(\hat{\theta})}{\gamma}
$$

and substituting the above solution and evaluating at $\hat{\theta}=\theta$ yields

$$
U_{\theta \theta}=-\frac{2 \beta^{2}}{\left(\alpha \gamma-\beta^{2}\right) \gamma}<0
$$

\section{Comparing both incentives}

Starting with the first observation, the property of no distortion at the bottom for the $\theta$ types and no distortion at the top for $t$-types, implies for interior (relaxed) program solutions that $x_{r}(\theta)>x_{r}(t)$ for $\theta=t \rightarrow \underline{t}$ and that $x_{r}(t)>x_{r}(\theta)$ at least for $t=\theta$ close to $\bar{t}$. The relaxed program outcome results from an intersection of the left hand side $=$ derivative of the joint surplus with respect to the contracted activity $x$ with the agency costs at the right hand side. Comparing the left hand sides of the relaxed program 
conditions, $l^{t}:=q_{x}+V_{x}$ from(22) in terms of $t$ and $l^{\theta}:=q_{x}+W_{x}=q_{x}+t V_{x}$ from (35) implies $l^{t}<l^{\theta} \Longleftrightarrow t=\theta<1, l^{t}>l^{\theta} \Longleftrightarrow t=\theta>1$ and identical at $t=1$ since $V_{x}<0$.

The right hand sides of the relaxed program conditions, denoted $r^{t}$ from (22) and $r^{\theta}$ from (35), determine the distortions from the first bests due to agency costs,. At small values of $t=\theta, r^{t}>r^{\theta}$ since $r^{\theta}(\underline{\theta})=0$, which combined with $l^{t}<l^{\theta}$ implies a larger task, $x_{r}(\theta)>x_{r}(t)$ at least for $t=\theta \rightarrow \underline{t}$. For large values of $t=\theta$ the converse applies to the left and the right hand side such that $x_{r}(t)>x_{r}(\theta)$, at least for $t=\theta$ close to $\bar{t}$.

Substituting $x_{r}(t)$, the agency costs are higher for the $t$-types at $t=1$,

$$
r^{t}-\left.r^{\theta}\right|_{t=1}>0 \Longleftrightarrow a \gamma>\beta(1+b+\bar{t})=\beta(2+b+\Delta) .
$$

Therefore, if the observable output is sufficiently important such the inequality at the right hand side of (43) holds, then the average $\theta$-type is asked for a higher observable output $x$ than its counterpart $t=1$; inequality (43) is stronger than the one assumed in 3 and the one derived below in (44). Surprisingly, the average agent characterized by an intrinsic motive for the unobservable task is asked to produce a higher level of the observable output. However, one can construct examples of only weakly more important $x$-outputs that satisfy the assumption (12) yet the opposite inequality of (43) holds, e.g., setting $a=3 / 2$ in the example in Fig. 1 .

In order to verify the second observation of a wider variation in $x_{r}(t)$ than in $x_{r}(\theta)$, let us compare the (interior) maxima,

$$
x_{r}(\vec{t})-x_{r}(\underline{\theta})=\frac{\Delta(a \gamma-(2+b) \beta)}{\alpha \gamma-\beta^{2}}>0 \Longleftrightarrow a>\frac{(2+b) \beta}{\gamma} .
$$

Therefore, agents characterized by their work ethic have a higher maximum in $x$, $x_{r}(\vec{t})>x_{r}(\underline{\theta})$, iff $a$ is sufficiently large satisfying the inequality in (44). This condition is sharper than the Assumption 2 but it is met in the example in Fig. 1 and less than the one in (43). At the minima the opposite holds iff,

$$
x_{r}(\bar{\theta})-x_{r}(\underline{t})=\frac{\Delta(a(3-\Delta) \gamma-((3-\Delta) b+2(\Delta-1)) \beta)}{\left(\alpha \gamma-\beta^{2}\right)(1+\Delta)}>0 \Longleftrightarrow a>\frac{b}{\gamma}+\frac{2(1-\Delta) \beta}{(3-\Delta) \gamma} .
$$

This above critical and minimal level of $a$ is met due to Assumption 2 ensuring nonnegativity and monotonicity, since

$$
\frac{\beta(1+b)}{\gamma}-\left(\frac{b}{\gamma}+\frac{2(1-\Delta)}{(3-\Delta)} \frac{\beta}{\gamma}\right)=\frac{1+\Delta}{3-\Delta} \frac{\beta}{\gamma}>0
$$

Hence, $x_{r}(\bar{\theta})>x_{r}(\underline{t})$. As a consequence, given the assumptions of $(i)$ interior solutions (i.e., relaxed programs satisfying the monotonicity properties), and (ii) a sufficiently large weight of the observable output in the principal's objective such that inequality (44), which is slightly sharper than Assumption 2, holds, then

$$
\left[x_{r}(\bar{\theta}), x_{r}(\underline{\theta})\right] \sqsubset\left[x_{r}(\underline{t}), x_{r}(\bar{t})\right] ;
$$

i.e., agents characterized by their work ethic type t face a wider range of tasks (for the observable output). 


\section{Interaction in output instead of in the agent's cost function}

How would the main findings change if, instead, task interdependencies are reflected by the production function and the agent has two technologically independent cost functions for the two tasks? In particular, is it still possible to construct an observation equivalent presentation of both approaches (i.e., work ethic and intrinsic motivation)? Will the main differences between both approaches still exist?

\section{Assumptions}

$$
\begin{aligned}
q & =a x+b y+\xi x y, \\
V & =y-\frac{1}{2} \frac{\alpha e_{x}^{2}+\gamma e_{y}^{2}}{t}, \\
W & =\theta y-\frac{1}{2}\left(\alpha e_{x}^{2}+\gamma e_{y}^{2}\right) .
\end{aligned}
$$

Work ethic $(t)$

$$
\begin{aligned}
y(t, x) & =\frac{t}{\gamma}, \\
V & =\frac{1}{2}\left(\frac{t}{\gamma}-\frac{\alpha x^{2}}{t}\right), \\
x_{0} & =0,
\end{aligned}
$$

i.e., just the special case of $\beta=0$ of the case analyzed in the paper. Since the task $y$, which has an intrinsic value to the agent, is not affected by the prescription of $x$, this eliminates the multi-tasking problem as demanding a higher observable output has no effect on the unobservable task.

\section{Intrinsic motivation}

$$
\begin{aligned}
y(\theta, x) & =\frac{\theta}{\gamma}, \\
W(x, \theta) & =\frac{\theta^{2}}{2 \gamma}-\frac{\alpha}{2} x^{2}, \\
x_{0} & =0 .
\end{aligned}
$$

as above, just the special case for $\beta=0$ and again, $y$ is unaffected by the principal's demand for $x$.

\section{References}

Baker G (2002) Distortion and risk in optimal incentive contracts. J Hum Resour 37:728-751 Benabou R, Tirole J (2006) Incentives and prosocial behavior. Am Econ Rev 96:1652-1678 
Benabou R, Tirole J (2016) Bonus culture: competitive pay, screening, and multitasking. J Polit Econ 124:305-370

Bo C, Chen Y, Rietzke D Simple contracts under observable and hidden actions. Economic Theory. https://doi.org/10.1007/s00199-019-01187-7

Feltham GA, Xie J (1994) Performance measure congruity and diversity in multi-task principal/agent relations. Account Rev 69:429-453

Fudenberg Drew D, Tirole J (1992) Game theory, 2nd printing. MIT Press, Cambridge

Goes PB, Ilk N, Lin M, Zhao JL (2018) When more is less: field evidence on unintended consequences of multitasking. Manag Sci 64(7):3033-3054

Hans G (1998) On the equivalence of general and specific control in organizations. Manag Sci 44(5):730-737

Helm C, Wirl F (2014) The Principal-Agent Model with Multilateral Externalities: An Application to climate agreements. J Environ Econ Manag 67:141-154

Helm C, Wirl F (2016) Multilateral externalities: contracts with private information either about costs or benefits. Econom Lett 141:27-31

Hind B, Nassim B (2010) Understanding developers' motives in open source projects: a multi-theoretical framework. Commun Assoc Inf Syst 27:30

Holmstrom B, Milgrom P (1991) Multitask Principal-Agent Analyses: Incentive Contracts, Asset Ownership, and job design. J Law Econ Organ 7:24-52

Jullien B (2000) Participation constraints in adverse selection models. J Econ Theory 93:1-47

Kerschbamer R, Maderner N (1998) Are two a good representative for many? J Econ Theory 83:90-104

Kolbjørnsrud V (2016) Agency problems and governance mechanisms in collaborative communities. Strategic Org 1476127016653727

Kragl J, Schöttner A (2014) Wage floors, imperfect performance measures, and optimal job design. Int Econ Rev 55:525-550

Lakhani Karim R, Wolf Robert (2005) Why hackers do what they do: understanding motivation and effort in free/open source software projects. In: Joe F Brian F, Scott H, Lakhani KR (eds) Perspectives on free and open source software. Cambridge: MIT Press

Lazear EP (2000) Performance pay and productivity. J Polit Econ 90:1346-1361

Maggi G, Rodriguez-Clare A (1995) On countervailing incentives. J Econ Theory 66:238-263

Martimort D, Sand-Zantman W (2013) Solving the global warming problem: beyond markets, simple mechanisms may help! Can J Econ 46:361-378

Martimort D, Sand-Zantman W (2016) A mechanism design approach to climate agreements. J Eur Econ Assoc 14:669-718

Mirrlees JA (1971) An exploration in the theory of optimum income taxation. Rev Econ Stud 38:175-208

Mullen KJ, Frank RG, Rosenthal MB (2010) Can you get what you pay for? Pay-for-performance and the quality of healthcare providers. RAND J Econ 41:64-91

Nicholas TN (2012) Antifragile. Allen Lane, London

Schnedler W (2008) When is it foolish to reward for A while benefiting from B? J Labor Econ 26:595-619

Wirl F, Huber C (2005) Voluntary Internalisations facing the threat of a pollution tax. Rev Econ Des 9:337-362

Wirl Franz (2003) Are stars worth their pay? In: Günther F, Uschi Backes-Gellner MS, Staufenbiel JE (eds) Modern concepts of the theory of the firm. Springer, Berlin, pp 505-519

Publisher's Note Springer Nature remains neutral with regard to jurisdictional claims in published maps and institutional affiliations. 